\title{
SCATTERING OF SURFACE WAVES BY A HALF IMMERSED CIRCULAR CYLINDER IN FLUID OF FINITE DEPTH
}

\author{
BIRENDRANATH MANDEL \\ Department of Applied Mathematics \\ University College of Science \\ 92 A.P.C. Road, Calcutta, India 700009 \\ And \\ SUDIP KUMAR GOSWAMI \\ Department of Mathematics \\ Presidency College \\ Calcuttâ, India 700073 \\ (Received November 19, 1982)
}

ABSTRACT. A train of surface waves is normally incident on a half immersed circular cylinder in a fluid of finite depth. Assuming the linearized theory of fluid under gravity an integral equation for the scattered velocity potential on the half immersed surface of the cylinder is obtained. It has not been found possible to solve this in closed form even for infinite depth of fluid. Our purpose is to obtain the asymptotic effect of finite depth " $h$ " on the transmission and reflection coefficients when the depth is large. It is shown that the corrections to be added to the infinite depth results of these coefficients can be expressed as algebraic series in powers of $a / h$ starting with $(a / h)^{2}$ where "a" is the radius of the circular cylinder. It is also shown that the coefficients of $(a / h)^{2}$ in these corrections do not vanish identically.

KEY WORDS AND PHRASES. Scattering, surface waves, fluid of finite depth, half immersed circular cylinder, reflection and transmission coejficients, putential function, Green's theorem.

1980 MATHEMATICS SUBJECT CLASSIFICATION CODE. 76 B

1. INTRODUCTION.

This paper deals with the reflection and transmission of surface waves normally incident on an infinitely long cylinder with circular cross section of radius "a" and horizontal generators, half immersed in an ideal fluid of infinite horizontal extent but finite constant depth " $h$." The two-dimensional linearized theory of an ideal fluid under gravity is assumed to hold in the fluid region. The motion of fluid can then be taken as irrotational and can be described by velocity potential. The existence and uniqueness of solution for a partly immersed obstacle was discussed by 
vohn [1] in fluid of both infinite and finite depth, although a closed form solutior is apparently not obtainable.

Dean ard Ursell [ $2 j$ investigated the interaction of a fixed half immersed circular cylinder with a train of surface waves in a fluid of infinite depth. They expressed the potential in the fluid region as a sum of wave source potentials and multiple singularities at the center of the circle with unknown coefficients and finally obtained an infinite set of linear algebraic equations involving the unknown coefficients. This infinite set, was then soived numerically for particular values of wave number, and the reflection and transmission coefficients were then computed numerically. The present problem is a generalization of the above where the depth of fluid is taken into account. By the use of Green's integral thenrem the velocity potential at any point in the fluid region can be made to depend upon the scattered potential on the half-immersed surface of the cylinder. This potential can be shown to satisfy an integral equation of the second kind by another use of Green's theorem. We recall that in the plane vertical barrier problems in fluid of infinite depth, by the use of Green's theorem and applying boundary condition on the barrier, a singular integral equation of the first kind in the difference of potential across the barrier could be obtained and this could be solved in a closed form (see Goswami [3]). Then for finite depth of fluid a perturbation technique could be applied so as to obtain correction to the reflection and transmission coefficients for finite depth over their infinite depth values in the cases when the barrier is in the form of a submerged fixed vertical barrier (see Goswami [4]) and a submerged fixed vertical plate (see Goswami [5]). MEi and Black [6] considered scattering of surface waves by rectangular obstacles fixed at the bottom or at the free surface in a fluid of finite depth and applied variational techriques to obtain the reflection and transmission coefficients numerically. From their results it is not apparently possible to obtain the effect of finite depth. Macaskill [7j recently considered scattering of surface waves by a vertical barrier of arbitrary permeability in a fluid of finite depth. By the use of Green's theorem he reduced the problem to a set of integral equations which are then solved numerically by using a special decomposition of the finite depth source potential.

For obstacles of types other than vertical barriers, the corresponding problem is rather complicated. Evell the apparently simple case of a half immersed circular cylinder in fluid of infinite depth can no longer be solved in closed forms, al though asymptotic methods are available for both long and short waves. For short waves in a fluid of infinite depth Ursell [8] used an integral equation approach. In this case there is only one parameter to consider, viz. Ka where $k$ is the infinite depth wave number and "a" is the radius of the cylinder. But in the finite depth case there are two independent parameters out of $\mathrm{Ka}, \mathrm{Kh}, \mathrm{a} / \mathrm{h}$ instead of only one in the infinite depth case. In the present paper we take $K a$ to be moderate and consider $h$ to be large so that $K h$ is large and $a / h$ is small. If we want to deal with cases where $K$ is very large or $K$ is very small, then the present method will perhaps fail. Under this assumption, it is found that the finite depth corrections of transmission and reflection coefficients over their infinite depth values car be expressed 
as algebralc series in $a / h$ starting with $(a / h)^{2}$. It is also shown that the coefficients of $(a / h)^{2}$ in these series do not vanish identically. A similar conclusion was arrived at by Goswami ([4], [5]) when the obstacle is in the form of a submerged vertical barrier and plate in fluid of finite depth. However, no numerical result is presented here.

2. FORMU:ATION OF THE PROBLEM.

We will use rectangular Cartesian coordinates with origin or the undisturbed free surface and at the center of the circular cylinder of radius "a," the y-axis vertically downwards, the $z$-axis horizontal along the generators of the cylinder and the $x$-axis perpendicular to the $y z$-plane. Let " $h$ " be the depth of water below the free surface. Let a train of surface waves from positive infinity ( $x$ direction) be made incident normally on the cylinder. The motion of the fluid is irrotational and can be described by a velocity potential given by $\operatorname{Re}\left\{\phi(x, y) e^{-i \sigma t}\right\}$ where $\sigma$ is the angular frequency, the incoming wave being described by $\operatorname{Re}\left\{\phi_{i n c}(x, y) e^{-i \sigma t}\right\}$, since the motion is two-dimensional and independent of $z$. The time independent complex valued potential function $\Phi(x, y)$ satisfies the Laplace's equation throughout the fluid region, i.e.,

$$
\left(\frac{\partial^{2}}{\partial x^{2}}+\frac{\partial^{2}}{\partial y^{2}}\right) \Phi=0 \quad, 0<y<h \quad,|x|<\infty \quad, r<a,
$$

where $r^{2}=x^{2}+y^{2}$. The linearized boundary condition on the undisturbed free surface is

$\frac{\partial \phi}{\partial y}+k \Phi=0, y=0,|x|>a$,

where $k=\sigma^{2} / g, g$ being the acceleration due to gravity. On the bottom of the fluid, the normal component of velocity is zero, so that

$$
\frac{\partial \Phi}{\partial y}=0, y=h \text {. }
$$

Also since the cylinder is at rest, there is no flow across the surface, so that

$$
\frac{\partial \Phi}{\partial r}=0 \text { on } r=a, y>0 \text {. }
$$

At great distance from the cylinder, the incident wave train produces a transmitted wave train moving in the negative $x$-direction for $x \rightarrow-\infty$ and a reflected wave train moving in the positive $x$ direction for $x \rightarrow+\infty$. These conditions can be mathematically expressed as

and

$$
\begin{array}{ll}
\Phi(x, y) \rightarrow \phi_{\text {inc }}(x, y)+R \phi_{\text {inc }}(-x, y) & \text { as } x \rightarrow+\infty \\
\Phi(x, y) \rightarrow T_{\text {inc }}(x, y) & \text { as } x \rightarrow-\infty
\end{array}
$$

where $R$ and $T$ are respectively the reflection and transmission coefficients which are to be determined.

The incident field is given by

$$
\phi_{\text {inc }}(x, y)=\frac{\cosh k_{0}(h-y)}{\cosh k_{0} h} e^{-i k_{0} x}
$$

where $k_{0}$ is the positive root of the transcendental equation

$$
k=k_{0} \tanh k o h \text {. }
$$

We note that, for large $h$,

$$
k_{0} \simeq k\left(1+2 e^{-2 k h}\right) \text {, }
$$

so that $k_{0}$ and $k$ differ by on exponentially small quantity for large $h$. 
Let us write

$$
\Phi(x, y)=\phi(x, y)+\phi_{\text {inc }}(x, y),
$$

so that $\phi(x, y)$ is the scattered potential and it satisfies $(2.1),(2.2),(2.3)$ together with

$$
<a \frac{\partial}{\partial r} \phi(x, y)>=-<a \frac{\partial}{\partial r} \phi_{\text {inc }}(x, y)>\equiv v(\theta) \text {, say, } 0<\theta<\pi
$$

where the angular bracket denotes the value at $r=a$, and $x=r \cos \theta, y=r \sin \theta$. Conditions $(2.5)$ and $(2.6)$ respectively reduce to

$$
\begin{array}{ll}
\phi(x, y) \rightarrow R \phi_{\text {inc }}(-x, y) & \text { as } x \rightarrow+\infty \\
\phi(x, y) \rightarrow(T-1) \phi_{\text {inc }}(x, y) & \text { as } x \rightarrow-\infty
\end{array}
$$

which show that $\phi(x, y)$ behaves as an outgoing wave as $|x| \rightarrow \infty$.

3. INTEGRAL EQUATION FOR $\phi(x, y)$ ON THE CYLINDER.

Let $G(x, y ; \xi, n)$ be the Green's function satisfying the Laplace equation

$\left(\frac{\partial^{2}}{\partial x^{2}}+\frac{\partial^{2}}{\partial y^{2}}\right) G=0$ except at $(\xi, n), 0<y<h,|x|<\infty$

with the conditions

$$
\begin{aligned}
& \frac{\partial G}{\partial y}+K G=0, \quad y=0, \quad|x|<\infty \\
& \quad \frac{\partial G}{\partial y}=0, \quad y=h, \quad|x|<\infty \\
& G+\ln \rho \text { as } \rho \equiv\left\{(x-\xi)^{2}+(y-n)\right\}^{\frac{1}{2}} \rightarrow 0,
\end{aligned}
$$

and

$$
G \rightarrow \text { a nultiple of } \frac{\cosh k_{0}(h-y)}{\cosh k_{0} h} e^{i k_{0}|x-\xi|} \quad \text { as }|x-\xi|+\infty .
$$

Condition (3.5) is the radiation condition at infinity. Then $G$ is given by (see Thorne [9])

$$
\begin{gathered}
G(x, y ; \xi, n)=\ln \frac{\rho}{\rho}-2 \oint_{0}^{\infty}\left(\frac{e^{-k h}}{k} \sinh k n \sinh k y+\right. \\
\frac{\cosh k(h-y) \cosh k(h-n)}{k \sinh k h-k \cosh k h} \frac{\cos k(x-\xi)}{\cosh k h} d k
\end{gathered}
$$

where $\rho^{\prime} \equiv\left\{(x-\xi)^{2}+(y+n)^{2}\right\}^{\frac{1}{2}}$, and the contour of integration is indented below the simple pole at $k=k_{0}$ to ensure the radiation condition (3.5) at infinity. For, as $|x-\xi| \rightarrow \infty$, it can be shown that

$$
G(x, y ; \xi, n) \rightarrow-2 \pi i F\left(k_{0} h\right) \frac{\cosh k_{0}(h-y) \cosh k_{0}(h-n)}{\cosh ^{2} k_{0} h} e^{i k_{0}|x-\xi|}
$$

where $F\left(k_{0} h_{1}\right)=\frac{2 \cosh ^{2} k_{0} h}{2 k_{0} h+\sinh 2 k_{0} h} \simeq 1+0\left(k h e^{-k h}\right.$; for large $h$.

Applying Green's integral theorem to $\phi(x, y)$ and $G(x, y ; a \cos \alpha$, a $\sin \alpha)$ in the fluid region with a small indentation at the point $(a \cos \alpha, a \sin \alpha)$ on the circle $r=a$, we obtain

$$
-\pi \phi(\alpha)+\int_{0}^{n} \phi(\theta)<a \frac{\partial}{\partial r} G_{1}(r, \theta ; a, \alpha)>d \theta=\int_{0}^{\pi} v(\theta)<G(r, \theta ; a, \alpha)>d \theta
$$


where $G_{1}(r, \theta ; a, \alpha)=G(x, y ; a \cos \alpha, a \sin \alpha)-\frac{1}{2} \ln \left[1(x-a \cos \alpha)^{2}\right.$

$\left.\left.+(y-a \sin \alpha)^{2}\right\} /\left\{(x-a \cos \alpha)^{2}+(y+a \sin \alpha)^{2}\right\}\right]$, and the angular brackets denote the values at $r=a$. Equation (3.9) provides ar integra? equation for $\phi(\theta)$ on the contour of the immersed portion of the cylinder. In deriving (3.9) we have made use of the fact that

$$
\int_{0}^{\pi}<a \frac{\partial}{\partial r} \ln \frac{(r \cos e-a \cos a)^{2}+(r \sin \theta-a \sin \alpha)^{2}}{(r \cos \theta-a \cos \alpha)^{2}+(r \sin e+a \sin \alpha)^{2}}>d \theta=0 .
$$

The poteritial function $\phi(\xi, n)$ at a general point $(\xi, n)$ of the fluid region can be obtained by applying Green's theorem in the fluid region to $\phi(x, y)$ and $G\left(x, y ; \xi, r_{1}\right)$, excluding the point $(\zeta, n)$, and is given by

$$
\begin{aligned}
-2 \pi \phi(\xi, n)=\int_{0}^{\pi} \phi(\theta)<a \frac{\partial}{\partial r} G(x, y ; \xi, n)>d \theta & \\
& \quad-\int_{0}^{\pi} v(\theta)<G(x, y ; \xi, n)>d \theta .
\end{aligned}
$$

Let the quantities witr. a superscript $\infty$ denote their infinite depth values. Then

$$
\begin{aligned}
G^{\infty}(x, y ; \xi, n) & =\ln \frac{\rho}{\rho^{\top}}-2 \oint_{0}^{\infty} \frac{e^{-k(y+n)}}{k-k} \cos k(x-\xi) d k \\
\phi_{\text {inc }}^{\infty}(x, y) & =e^{-k y-i k x} .
\end{aligned}
$$

Let

$$
G=G^{\infty}+G_{D}, \phi_{i n c}=\phi_{i n c}^{\infty}+\phi_{i n c}^{D}(x, y) .
$$

We note that $\phi_{\text {inc }}^{D}(x, y)$ is exponentially small for large $h$. Also, from (3.16) and (3.11), $G_{D}(x, y ; \xi, n)$ can readily be shcwn to be equal to

$-2 \oint_{0}^{\infty} \frac{e^{-k h}(k \sinh k y-k \cosh k y)(k \sinh k n-k \cosh k n)}{k(k-k)(k \sinh k h-k \cosh k h)} \cos k(x-\xi) d k$

where the contour is indented below the poles at $k=k$ and $k=k_{0}$. For infinite depth the integral equation (3.9) reduces to

$$
\begin{gathered}
-\pi \phi^{\infty}(\alpha)+\int_{0}^{\pi} \phi^{\infty}(\theta)<a \frac{\partial}{\partial r} G_{1}^{\infty}(r, \theta ; a, \alpha)>d \theta \\
=\int_{c}^{\pi} v^{\infty}(\theta) G^{\infty}(a, \theta ; a, \alpha) d \theta, 0<\alpha<\pi .
\end{gathered}
$$

Let

where obviously $v_{D}(\theta)$ is exponentially small for large $h$, and

$$
\phi(\theta)=\phi^{\infty}(\theta)+\phi_{D}(\theta), \quad v(\theta)=v^{\infty}(\theta)+v_{D}(\theta)
$$

$$
v^{\infty}(\theta)=-<a \frac{\partial}{\partial r} \phi_{i n c}^{\infty}(x, y)>=i K a e^{-i K a \cos \theta-K a \sin \theta-i \theta} \text {. }
$$

From (3.9) using (3.14) and neglecting exponentially small quantities for large $h$, we obtain the integral equation for $\phi_{D}(\theta)$ as

$-\pi \phi_{D}(\alpha)+\int_{0}^{\pi} \phi_{D}(\theta)\left\{<a \frac{\partial}{\partial r} G_{1}^{\infty}(r, \theta ; a, \alpha)>+<a \frac{\partial}{\partial r} G_{D}(r, \theta ; a, \alpha)>\right\} d \theta$ 


$$
\begin{aligned}
=\int_{0}^{\pi} & v^{\infty}(\theta)<G_{D}(r, \theta ; a, \alpha)>d \theta \\
& \quad-\int_{0}^{\pi} \phi^{\infty}(\theta)<a \frac{\partial}{\partial r} G_{D}(r, \theta ; a, \alpha)>d \theta, 0<\alpha<\pi
\end{aligned}
$$

where now $\phi^{\infty}(\theta)$ is supposed to be known being obtained as the solution of the integral equation (3.14).

4. REFLECTION AND TRANSMISSION COEFFICIENTS.

Using (3.7) in (3.10) we obtain as $\xi \rightarrow \infty$,

$$
\begin{aligned}
\phi(\xi, n) \rightarrow F\left(k_{0} h\right) \phi_{i n c}(-\xi, n) \int_{0}^{\pi}\left[\phi(\theta) a k_{0} \cosh \left\{k_{0}(h-a \sin \theta)-i \theta\right\}\right. \\
\left.-i v(\theta) \cosh k_{0}(h-a \sin \theta)\right] \frac{e^{-i k_{0} a \cos \theta}}{\cosh k_{0} h} d \theta
\end{aligned}
$$

so that by (2.12) the reflection coefficient $R$ is given by,

$$
\begin{aligned}
R=F\left(k_{0} h\right) & \int_{0}^{\pi}\left[\phi(\theta) a k_{0} \cosh \left\{k_{0}(h-a \sin \theta)-i \theta\right\}\right. \\
& \left.-i v(\theta) \cosh k_{0}(h-a \sin \theta)\right] \frac{e^{-i k_{0} a \cos \theta}}{\cosh k_{0} h} d \theta .
\end{aligned}
$$

Again, similarly using (3.7) in (3.10) we obtain as $\xi \rightarrow-\infty$,

$$
\begin{gathered}
\phi(\xi, n) \rightarrow-F\left(k_{0} h\right) \phi_{i n c}(\xi, n) \int_{0}^{\pi}\left[\phi(\theta) \alpha k_{0} \cosh \left\{k_{0}(h-a \sin \theta)+i \theta\right\}\right. \\
\left.+i v(\theta) \cosh k_{0}(h-a \sin \theta)\right] \frac{e^{i k_{0} a \cos \theta}}{\cosh k_{0} h} d \theta
\end{gathered}
$$

so that by (2.13), we obtain the transmission coefficient $T$ as

$$
\begin{aligned}
T=1-F\left(k_{0} h\right) & \int_{0}^{\pi}\left[\phi(\theta) a k_{0} \cosh \left\{k_{0}(h-a \sin \theta)+i \theta\right\}\right. \\
& \left.+i v(\theta) \cosh k_{0}(h-a \sin \theta)\right] \frac{e^{i k_{0} a \cos \theta}}{\cosh k_{0} h} d \theta .
\end{aligned}
$$

Making $h \rightarrow \infty$ in (4.1) we obtain

$$
R^{\infty}=a K \int_{0}^{\pi}\left[\phi^{\infty}(\theta) e^{-K a(\sin \theta+i \cos \theta)}\right] e^{-K a(\sin \theta+i \cos \theta)-i \theta} d \theta .
$$

Neglecting exponentially small quantities for large $h$ we obtain

$$
R-R^{\infty} \simeq a K \int_{0}^{\pi} \phi_{D}(\theta) e^{-K a(\sin \theta+i \cos \theta)-i \theta} d \theta \text {. }
$$

Sinilarly from (4.2)

$T^{\infty}=1-a K \int_{0}^{\pi}\left[\phi^{\infty}(\theta) e^{i \theta}-e^{-K a(\sin \theta-i \cos \theta)-i \theta}\right] e^{-K a(\sin \theta-i \cos \theta)} d \theta$,

and neglecting exponentially small quantities

$$
T-T^{\infty} \simeq-a K \int_{0}^{\pi} \phi_{D}(\theta) e^{-K a(\sin \theta-i \cos \theta)+i \theta} d \theta \text {. }
$$

Thus the depth correction to the reflection and transmission coefficients over their infinite depth values are approximately given by (4.4) and (4.6) respectively for large $h$. It will be shown that these are algebraically small for large $h$ and, in fact, can be expressed as algebraic series in a/h starting with $(a / h)^{2}$. The 
quantity $\phi_{D}(\theta)$ in (4.4) and (4.6) satisfies the integral equation (3.16). Now $<G_{D}>$ and $<a \frac{\partial}{\partial r} G_{D}(r, \theta ; a, \alpha)>$ in the right side of $(3.16)$ can be expressed as a series in terms of $a / h$ for large $h$ starting with $(a / h)^{2}$ assuming $\mathrm{Ka}$ to be fixed. This suggests that $\phi_{D}(\theta)$ has also a similar type of expansion for large $h$. Thus the expressions (4.4) ar.d (4.6) are algebraically small for large $h$. That the first terms in the expansion of (4.4) and (4.6) are nonvanishing will be shown later. These are done in the next sections.

5. ASYMFTOTIC EXPANSION FOR LARGE $h$.

By (3.13)

$G_{D}(x, y ; c, n)=-2 \int_{0}^{\infty} \frac{e^{-k h}(k \sinh k y-k \cosh k y)(k \sinh k n-k \cosh k n)}{k(k-k)(k \cosh k h-k \sinh k h)} \operatorname{cosk}(x-\xi) d k$ (5.1)

In view of (3.16), we confine our attention to $|y| \leq a,|n| \leq a,|x| \leq a,|\xi| \leq a$. The integrand in (5.1) (excepting the term $\cos k\left(x-\xi_{3} ;\right.$ ) is of the order $e^{-2 k h} e^{2 k a}$, which is obviously expcrentially small when $k>k^{*}$ where $k^{*} x$ sufficiently large, and it is still exponentially small when we replace |cos $k\left(x-s_{1}\right)$ ! by the crude tound $\cos 2 \mathrm{ka}$. Thus we can expand the factors in

$$
\frac{(k \sinh k y-k \cosh k y)\left(k \sinh k n-k \cosh k r_{1}\right)}{k(k-k)} \cos k(x-\xi)
$$

by the power series expansion in $k$. Also write

$$
\begin{gathered}
\frac{e^{-k h}}{k \cosh k h-k \sinh k h}=\frac{e^{-k h}}{k \cosh k h} \sum_{m=0}^{\infty}\left(\frac{k}{k}\right)^{m} \operatorname{tar}^{m} h^{m} k h \\
=\frac{1}{K}(1-\tanh k h) \sum_{m=0}^{\infty}\left(\frac{k}{k}\right)^{m} \tanh ^{m} k h .
\end{gathered}
$$

In (5.2), put $k=K u$, then (5.2) becomes

$$
\begin{gathered}
\frac{(K \sinh u K y-K u \cosh u K y)(K \sinh u K n-K u \cosh K u n) \cos u(K x-K \xi)}{u K^{2}(1-u)} \\
=\sum_{s=1}^{\infty} u^{s} f_{s}(K y, K n, K|x-\xi|) \text {, say, }
\end{gathered}
$$

where $f_{1}\left(K y, K r_{1}, K|x-\xi|\right)=(K y-1)(K n-1)$,

and other $f_{s}^{\prime} s$ can be found.

Thus the integrand in (5.1) can be expanded as

$$
\frac{e^{-k h}}{k \cosh k h}\left(\sum_{m=0}^{\infty}\left(\frac{k}{k}\right)^{m} \tanh ^{m} k h\right)\left(\sum_{s=1}^{\infty}\left(\frac{k}{k}\right)^{s} f_{s}(k y, k n, k|x-\xi|)\right) .
$$

Hence $G_{D}(x, y ; \xi, n)=-2 \int_{0}^{\infty} \frac{e^{-u}}{k \cosh u}\left(\sum_{m=0}^{\infty} \frac{u^{m} \tanh ^{m} u}{(K h)^{m}}\right)\left(\sum_{s=1}^{\infty} \frac{u^{s}}{(K h)^{s}} x\right.$

$$
=-\frac{2}{K h} \sum_{m=0}^{\infty} \sum_{s=1}^{\infty} \frac{1}{(K h)^{m+s}} f_{s}(K y, K n, K|x-\xi|) a_{m s}
$$

where

$$
o_{m s}=\int_{0}^{\infty} \frac{e^{-u}}{\cosh u} u^{m+s} \tanh ^{m} u d u \text {. }
$$

Thus the first term in the expansion of $G_{D}(a \cos e, a \sin \theta ; a \cos \alpha, a \sin \alpha)$ is

$-\frac{2 \alpha_{01}}{(K h)^{2}} f_{1}(K a \sin \theta, K a \sin \alpha, K a|\cos \theta-\cos \alpha|)=-\left(\frac{a}{h}\right)^{2} \frac{2 \alpha_{01}}{(K a)^{2}}(K a \sin e-1)(K a \sin \alpha-1)$ 
and the first term in the expansion of $<a \frac{\partial}{\partial r} G_{D}(r \cos \theta, r \sin \theta ; a \cos \alpha, a \sin \alpha)=$ is

$$
\begin{aligned}
& -\frac{2 \alpha_{01}}{(K h)^{2}}<a \frac{\partial}{\partial r} f_{1}(K . y, K a \sin \alpha, K|x-a \cos \alpha|)> \\
= & -\frac{2 K a \alpha_{01}}{(K h)^{2}}(K a \sin \alpha-1) \sin \theta=-\left(\frac{a}{h}\right)^{2} \frac{2 \alpha_{01}}{K a}(K a \sin \alpha-1) \sin \theta,
\end{aligned}
$$

where

$$
a_{01}=\int_{0}^{\infty} \frac{e^{-u}}{\cosh u} u d u=\pi^{2} / 24 \text {. }
$$

These suggest an asymptotic expansion of $\phi_{D}(\theta)$ for large " $h "$ as

$$
\phi_{D}(\theta)=\frac{1}{(K h)^{2}} \psi_{D}(\theta)+\frac{1}{(K h)^{3}} \psi_{D}^{(3)}(\theta)+\ldots,
$$

when ${ }_{C}(\theta)$ satisfies

$$
\begin{aligned}
& -\pi \psi_{D}(\alpha)+\int_{0}{ }^{\pi} \psi_{D}(\theta)<a \frac{\partial}{\partial r} G_{1}^{\infty}(r \cos \theta, r \sin \theta ; a \cos \alpha, a \sin \alpha)>d \theta \\
& =-2 \alpha_{01}(K a \sin \alpha-1) 0_{0} \cdot{ }^{\pi}\left\{v^{\alpha}(\theta)(K a \sin \theta-1)-K a \phi^{\infty}(\theta) \operatorname{sir} \theta\right\} d \theta
\end{aligned}
$$

where $\phi^{\infty}(\theta)$ in the right side is known in principle. Thus to a first approximation

$$
\left(R-R^{\infty}\right)_{1}=\left(\frac{a}{h}\right)^{2} \frac{1}{K_{a}} \int_{0}^{\pi} \psi_{0}(\theta) e^{-K a(\sin \theta+i \cos \theta)-i \theta} d \theta
$$

and

$$
\left(T-T^{\infty}\right)_{1}=-\left(\frac{a}{h}\right)^{2} \frac{1}{K a} c^{S^{\pi}} \psi_{D}(\theta) e^{-K a(\sin \theta-i \cos \theta)+i \theta} d \theta .
$$

We will now show that the integrals in (5.4) do not vanish identically. These integrals can be regarded as functions of $\mathrm{Ka}$ regular for all values of $\mathrm{Ka}$. As these integrals no longer involve $h$, we can waive the restriction to Ka originally imposed and instead we assume $K a$ to be small in the discussion of the following section.

6. APPROXIMATE VALUES FOR $\phi^{\infty}(\theta)$.

In the above we have stated that $\phi^{\infty}(\theta)$ is known in principle which is in fact the solution of the integral equation (3.14). As it is almost impossible to solve analytically this integral equation for any $\mathrm{Ka}$, without any loss of generality we will assume that $\mathrm{Ka}$ is small, and then obtain an approximate value of $\phi^{\infty}(\theta)$. Using this we will show that the integrals in (5.4) do not vanish for small $\mathrm{Ka}$, and hence these do not identically vanish for any $\mathrm{Ka}$.

Following Ursell [8], we can show that

$$
\begin{aligned}
& G^{\infty}(a \cos \theta, a \sin \theta, a \cos \alpha, a \sin \alpha)=\ln \left|\frac{\sin \frac{\theta-\alpha}{2}}{\sin \frac{\theta+a}{2}}\right| \\
&+2\left[\left\{(\gamma+\ln 2 k a-\pi i)+\ln \sin \frac{e+a}{2}\right\}<e^{-K(y+n)} \cos K(x-\xi)>\right. \\
&\left.-\frac{\alpha-\theta}{2}<e^{-K(y+n)} \sin K(x-\xi)>+\sum_{m=1}^{\infty}(-1)^{m-1} \frac{\left(1+\frac{1}{2}+\ldots+\frac{1}{m}\right)}{m !}\left(2 K a \sin \frac{\theta+\alpha}{2}\right)^{m} \operatorname{cosm} \frac{\alpha-\theta}{2}\right]
\end{aligned}
$$


and

$<a \frac{\partial}{\partial r} G_{1}^{\infty}(r \cos \theta, r \sin \theta ; a \cos \alpha, a \sin \alpha)>=\left\langle e^{-K(y+n)} \cos K(x-\xi)\right\rangle+$

$$
\begin{aligned}
& 2\left[i(r+\ln 2 K a-\pi i)+\ln \sin \frac{\theta+2}{2}\right\}<a \frac{\partial}{\partial r} e^{-K(y+n)} \cos K(x-\xi)> \\
- & <\zeta a \frac{\partial}{\partial r} e^{-K(y+n)} \sin k(x-\xi)> \\
+ & \left.\sum_{m=1}^{\infty}(-1)^{m-1} \frac{\left(1+\frac{1}{2}+\ldots+\frac{1}{m}\right)}{m !}<a \frac{\partial}{\partial r}(K p)^{m} \cos m \zeta>\right]
\end{aligned}
$$

where $\xi=a \cos \alpha, n=a \sin \alpha,\left\langle\zeta>=\frac{u-\theta}{2},<\rho>=2 a \sin \frac{\theta+\alpha}{2}\right.$, and the angular brackets denote the values for $r=a$.

The integral equation (3.14) can now be rewritten as

$-\pi \phi^{\infty}(\alpha)+\int_{0}^{\infty} M(\theta, \alpha) \phi^{\infty}(\theta) d \theta=L(a, K a)-A(K a)<e^{-k n} \cos K \xi>$

$$
-B(K a)<e^{-k r_{1}} \sin K \xi>, 0<\alpha<\pi \text {, }
$$

where

$$
\begin{aligned}
M(\theta, a)=2\left[\ln \sin \frac{\theta+\alpha}{2}\right. & <\mathrm{a} \frac{\partial}{\partial r} e^{-k(y+n)} \cos K(x-\xi)>-\left\langle\zeta \text { a } \frac{\dot{\partial}}{\partial r} e^{-K(y+n)} \sin k(x-\xi)>\right. \\
& \left.+\sum_{m=1}^{\infty}(-1)^{m-1} \frac{\left(1+\frac{1}{2}+\ldots \frac{1}{}+{ }_{m}\right)}{m !}<a \frac{\partial}{\partial r}(K \rho)^{m} \cos m \zeta>\right], \quad(6.4)
\end{aligned}
$$

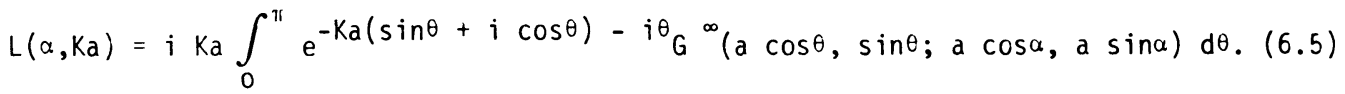
$\begin{aligned} & A \\ & B\end{aligned}=\int_{0}^{\pi} \phi^{\infty}(\theta)<e^{-k y} \cos k x>+2(\gamma+\ln 2 k a-\pi i) \int_{0}^{\pi} \phi^{\infty}(\theta)$

$$
<a \frac{\partial}{\partial r} e^{-k y} \cos k x>d \theta
$$

Neglecting terms of order $O\left(\left(K_{a}\right)^{2}\right.$ in $\left.K a\right)$ we see that

$$
M(\theta, \alpha)=K a M_{1}(\theta, \alpha)+0(K a)^{2}
$$

where

$$
M_{1}(\theta, \alpha)=-2 \ln \sin \frac{\theta+\alpha}{2} \sin \theta-(\alpha-\theta) \cos \theta+(\sin \theta+\sin \alpha)
$$

so that $M_{1}(\theta, \alpha)$ is bounded for all values of $\theta, \alpha$ in $(0, \pi)$. Also

$$
L(\alpha)=K a L_{1}(\alpha)+0\left((K a)^{2} \ln K a\right)
$$

where $L_{1}(\alpha)=4(\gamma+\ln 2 K a-\pi i)-2 \ln 2-2+2 \ln \sin \alpha$

$$
-2 \cos \alpha \ln \tan \frac{\alpha}{2}-\pi i \cos \alpha \text {. }
$$

Thus $L_{1}(\alpha)$ is bounded for all values of $\alpha$ in $(0, \pi)$. For the time being we assume that $A(\mathrm{Ka})$ and $B(\mathrm{Ka})$ are known. We now apply the following theorem due to Ursell [8] to the integral equation (6.3).

Theorem If $(i) \mathrm{q}(\alpha)$ is bounded in $(0, \pi)$

$$
\text { (ii) } \frac{1}{\pi} \int_{0} \int^{\pi}|L(\theta, \alpha)| d \theta \leq m<1,0<\alpha<\pi \text {, }
$$

then the integral equation

$$
\pi p(\alpha)-\int_{0}^{\pi} L(\theta, \alpha) p(\theta) d \theta=q(\alpha), 0<\alpha<\pi,
$$


may be solved by iteration, and

$$
p(\alpha)=q(\alpha)+\sum_{s=1}^{\infty} q_{s}(\alpha)
$$

where

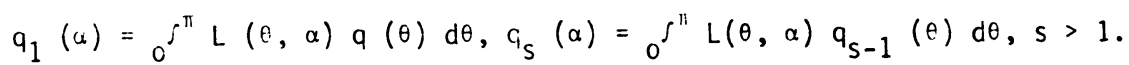

The applicability of the theorem to the integral equation (6.3) is assured by the fact that by virtue of $(6.7)$, the condition ( $i \mathrm{i}$ ) is satisfied for small $\mathrm{Ka}$, and by virtue of (6.8), the conditiori ( $i$ ) is satisfied for small $\mathrm{Ka} ; A(K a)$ and $B(K a)$ are assumed to be such that the whole of the right side of (6.3) satisfies the condition (i) of the theorem.

Now the integral equation (6.3) can symbolically be written as

$$
-\pi\left(I-\frac{1}{\pi} M\right) \phi^{\infty}(\alpha)=L(\alpha)-A<e^{-K n} \cos K \xi>-B<e^{-K n} \operatorname{sinK\xi }>
$$

so that

$$
-\pi \phi^{\infty}(\alpha)=\left(I-\frac{1}{\pi} M\right)^{-1} L-A\left(I-\frac{1}{\pi} M\right)^{-1}<e^{-K n} \cos K \xi>
$$

$$
-B\left(1-\frac{1}{\pi} M\right)^{-1}<e^{-K n} \operatorname{sinK\xi }>
$$

where I denotes the identity operator. Neglecting terms of the order of $0\left((K a)^{2}\right) \quad 0\left((K a)^{2} \ln K a\right)$, we see that

the first term in $(6.9)=K a L_{1}(\alpha)$

the second term in $(6.9)=1-K a \sin \alpha+\frac{1}{\pi} K a\left(2+2 \ln 2-2 \ln \sin \alpha+2 \cos \alpha \ln \tan \frac{\alpha}{2}\right)$ the third term in (6.9) $=K a \cos \alpha$.

Thus

$-\pi \phi^{\infty}(\alpha)=K a L_{1}(\alpha)-A\left\{1-K a \sin \alpha+\frac{1}{\pi} K a\left(2+2 \ln 2-2 \ln \sin \alpha+2 \cos \alpha \ln \tan \frac{\alpha}{2}\right)\right\}$

$$
\text { - B Ka } \cos \alpha+O\left((K a)^{2},(K a)^{2} \ln K a\right) \text {. }
$$

Now the equations for the determination of $A$ and $B$ are obtained by using (6.9) in (6.6). These equations are

$$
\begin{aligned}
& A\left[-\pi+\int_{0}^{\pi}\left\{\left(I-\frac{1}{\pi} M\right)^{-1}<e^{-K n} \cos K \xi>\right\}<e^{-K y} \cos K x>d \theta\right. \\
& \left.\quad+2(r+\ln 2 K a-\pi i) \int_{0}^{\pi}\left\{\left(I-\frac{1}{\pi} M\right)^{-1}<e^{-K n} \cos K \xi>\right\}<a \frac{\partial}{\partial r} e^{-K Y} \cos K x>d \theta\right] \\
& +B\left[\int_{0}^{\pi}\left\{\left(I-\frac{1}{\pi} M\right)^{-1}<e^{-K n} \operatorname{sinK\xi }>\right\}<e^{-K y} \cos K x>d \theta\right. \\
& \left.\quad+2(\gamma+\ln 2 K a-\pi i) \int_{0}^{\pi}\left\{\left(I-\frac{1}{\pi} M\right)^{-1}<e^{-K \eta} \operatorname{sinK\xi }>\right\}<a \frac{\partial}{\partial r} e^{-K y} \cos K x>d \theta\right] \\
& =\int_{0}^{\pi}\left\{\left(I-\frac{1}{\pi} M\right)^{-1} L(\alpha)\right\}<e^{-K y} \cos K x>d \theta \\
& \quad+2(r+\ln 2 K a-\pi i) \int_{0}^{\pi}\left\{\left(I-\frac{1}{\pi} M\right)^{-1} L(\alpha)\right\}<a \frac{\partial}{\partial r} e^{-K y} \cos K x>d \theta,
\end{aligned}
$$

and 


$$
\begin{aligned}
& A\left[\int_{0}^{\pi}\left\{\left(I-\frac{1}{\pi} M\right)^{-1}<e^{-K n} \cos K \xi>\right\}<e^{-K y} \operatorname{sinKx}>d \theta\right. \\
& \left.\quad+2(r+\ln 2 K a-\pi i) \int_{0}^{\pi}\left\{\left(I-\frac{1}{\pi} M\right)^{-1}<e^{-K n} \cos K \xi>\right\}<a \frac{\partial}{\partial r} e^{-K y} \operatorname{sinKx}>d \theta\right] \\
& +B\left[-\pi+\int_{0}^{\pi}\left\{\left(I-\frac{1}{\pi} M\right)^{-1}<e^{-K r_{1}} \operatorname{sinK} \xi>\right\}<e^{-K y} \operatorname{sinKx}>d \theta\right. \\
& \quad+2(\gamma+\ln 2 K a-\pi i) \int_{0}^{\pi}\left\{\left(I-\frac{1}{\pi} M\right)^{-1}<e^{-K n} \operatorname{sinK\xi }>\right\}<a \frac{\partial}{\partial r} e^{-K y} \operatorname{sinKx}>d \theta \\
& =\int_{0}^{\pi}\left\{\left(1-\frac{1}{\pi} M\right)^{-1} L(\alpha)\right\}<e^{-K y} \operatorname{sinKx}>d \theta \\
& \quad+2(r+\ln 2 K a-\pi i) \int_{0}^{\pi}\left\{\left(I-\frac{1}{\pi} M\right)^{-1} L(\alpha)\right\}<a \frac{\partial}{\partial r} e^{-K y} \operatorname{sinKx}>d \theta
\end{aligned}
$$

where in the terms in all the second brackets $\alpha$ is to be replaced by $\theta$. From (6.11) we obtain

$$
\begin{aligned}
A\{-4 K a(1+\gamma+\ln K a-\pi i)+ & \left.0\left((K a)^{2} \operatorname{ln~Ka,}(K a)^{2}\right)\right\}+B\left\{0\left((K a)^{2}\right)\right\} \\
= & \pi K a\{4(\gamma+\ln K a-\pi i)\}+0\left((K a)^{2}\right)
\end{aligned}
$$

so that

$$
A(K a)=A_{0}+0(K a \ln K a)
$$

where $A_{0}=-\pi+\frac{\pi}{1+\gamma+\operatorname{ln~Ka}-\pi i}$.

We note that it is sufficient to determine $A(K a)$ up to this order. In (6.12), the coefficent of $A$ is $0\left((K a)^{3}\right)$ and the coefficient of $B$ is

$$
\pi\left\{-1+\frac{(K a)^{2}}{2}+(K a)^{2}(\gamma+\ln 2 K a-\pi i)+0\left((K a)^{3} \ln K a\right)\right\}
$$

while the right side of $(6.12)$ is

Hence $B(K a)=0\left((K \cdot a)^{2}\right)$, and we neglect it compared to $A$.

Thus

$$
\begin{aligned}
-\pi \phi^{\infty}(\alpha)=K a L_{1}(\alpha)- & A_{0}\left[1+K a\left\{-\sin \alpha+\frac{2}{\pi}(1+\ln 2-\ln \sin \alpha\right.\right. \\
& \left.\left.\left.+\cos \alpha \ln \tan \frac{\alpha}{2}\right)\right\}\right]+0\left((K a)^{2} \ln K a\right)
\end{aligned}
$$

where $A_{0}$ is given by $(6.13)$. Hience $\phi^{\infty}(\alpha)$ is approximated for small Ka.

We next consider the integral equation (5.3) for $\Psi_{D}(\alpha)$.

This can be rewritten as

$$
\begin{aligned}
-\pi \psi_{D}(\alpha)+\int_{0}^{\pi} \psi_{D}(\theta) M(\theta, \alpha) d \theta= & L_{D}(\alpha)-A_{D}(K a)\left\langle e^{-K n} \cos K \xi>\right. \\
& -B_{D}(K a)<e^{-K n} \sin K \xi>
\end{aligned}
$$

where

$L_{D}=-2 K a \alpha_{01}(K a \sin \alpha-1)\left[i \int_{0}^{\pi} e^{-K a(\sin \theta+i \cos \theta)-i \theta}(K a \sin \theta-1) d \theta\right.$

$$
\left.-\int_{0}^{\pi} \phi^{\infty}(\theta) \sin \theta d \theta\right]
$$




$$
\begin{aligned}
& { }_{D}^{A_{D}}=\int_{0}^{\pi} \psi_{D}(\theta)<e^{-K y} \cos \sin K x>d \theta+ \\
& 2(r+\ln 2 K a-i \pi) \int_{0}^{\pi} \psi_{D}(\theta)<a \frac{\partial}{\partial r} e^{-K y \cos } \sin K x>d \theta .
\end{aligned}
$$

We note that

$$
L_{D}=-\frac{4 u_{01} K a}{1+\gamma+\ln K a-\pi i}+0\left((K a)^{2} \ln K a\right) .
$$

Proceeding as in the case of $\phi^{\infty}$, we obtain

$$
\begin{aligned}
& A_{D}=A_{D O}+0(K a \ln K a) \\
& E_{D}=0\left((K a)^{2} \ln K a\right)
\end{aligned}
$$

where

$$
A_{D O}=\frac{\pi \alpha_{01}}{(1+\gamma+\ln K a-\pi i)^{2}}
$$

and

$$
-\pi \psi_{D}=-\frac{4 \alpha_{01} K a}{1+\gamma+\ln K a-\pi i}-A_{D O}\left[1+\frac{0(K a \ln K a)}{1+\gamma+\ln K a-\pi i}\right] .
$$

Hence

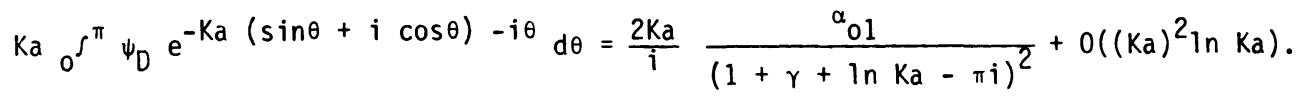

Thus for small $\mathrm{Ka}$,

$$
\left.\begin{array}{l}
\left(R-R^{\infty}\right)_{1}=-\frac{1}{(K h)^{2}}\left[\frac{2 i \alpha_{01} K a}{(1+\gamma+\ln K a-\pi i)^{2}}+0\left((K a)^{2} \ln K a\right)\right] \\
\left(T-T^{\infty}\right)_{1}=-\frac{1}{(K h)^{2}}\left[\frac{2 i \alpha_{01} K a}{(1+\gamma+\ln K a-\pi i)^{2}}+0\left((K a)^{2} \ln K a\right)\right]
\end{array}\right\}
$$

Thus for small Ka, the coefficients $\left(R-R^{\infty}\right)_{1},\left(T-T^{\infty}\right)_{1}$ of $(a / h)^{2}$ in the expansion of $R-R^{\infty}$ and $T-T^{\infty}$ respectively are not zero, and hence these do not vanish identically.

7. CONCLUSION.

The depth effect on the reflection and transmission coefficients for the case of a normally incident wave on a half inmersed circular cylinder is not toc small to be neglected. If however, the depth is assumed to be large (but not infinite), then within the framework of our above analysis, the depth corrections to these coefficients over their infinite depth values, can be expressed as algebraic series in powers of $a / h$ commencing with $(a / h)^{2}$.

\section{ACKNOWLEDGEMENT}

One of the authors ( $S K G$ ) thanks the U.G.C., India, for the award of a Teacher Fellowship under the Faculty Improvement scheme. 


\section{inLI LRENCES}

1. JOHN, F. On the motion of floating bodies II, Commin. Pure Appl. Math. 3 , (1950), $45-101$.

2. DEAN, R. G. and URSELL, F. Interaction of a Semi-immersed circular cylincer with a train of surface waves, Hydrcciynamics Laboratory, repartment cf Civil and Sanitary Engineering, MIT, (1959).

3. GOSWAMI, S. K. A note on thic problem of scattering cif surface waves by a subnerged fixed vertical tiarrier, ZAMM 62 (1982), 637-639.

4. GOSWAMI, S. K. Diffraction of rater waves ty a submerced fixed vertical barrier in viater of finite depth, Eull. Cal. Math. Soc., 74, (198ï), 75-86.

5. GOSWAMI, S. K. Scattering of surface waves by a submerged fixed vertical plate in water of finite depth, J. Indian Inst. Sci. (198,2), 79-88.

6. MEI, C. C. and BLACK, J. L. Scattering of surface waves bi rectangular c.tstacles in waters of finite depth, J. Fluid Mech. 38, (1969), 499-511.

7. MACASKILL, C. Reflexion of water waves by a perneable barrier, J. Fluid Mech. 95, (1979), 141-157.

8. URSELL, $F$. The transmission of surfoce waves under surface obstacles, Proc. Camb. Philos. Soc. 57 (1961), 638-663.

9. THORNE, R. C. Multipole expansions in the theory of surface waves, Proc. Camb. Philos. Soc. 49, (1953), 707-716. 


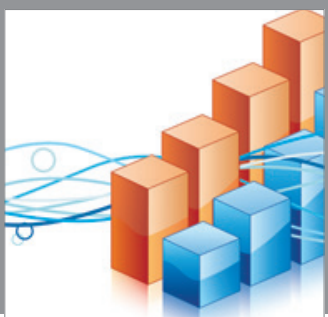

Advances in

Operations Research

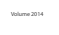

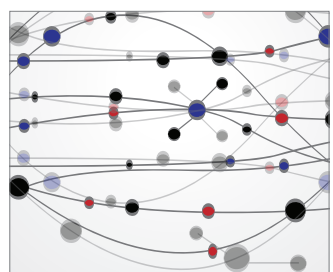

\section{The Scientific} World Journal
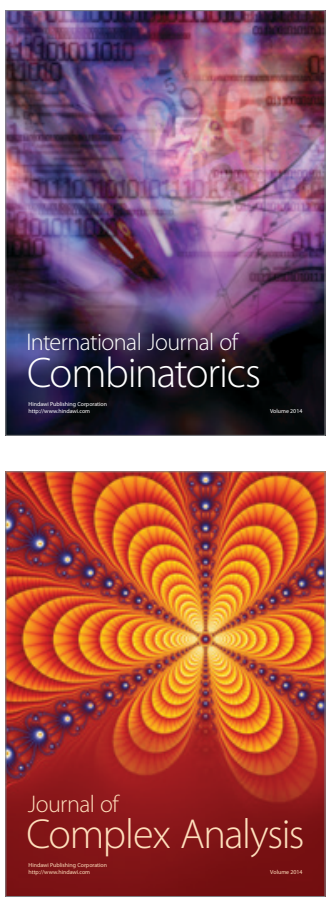

International Journal of

Mathematics and

Mathematical

Sciences
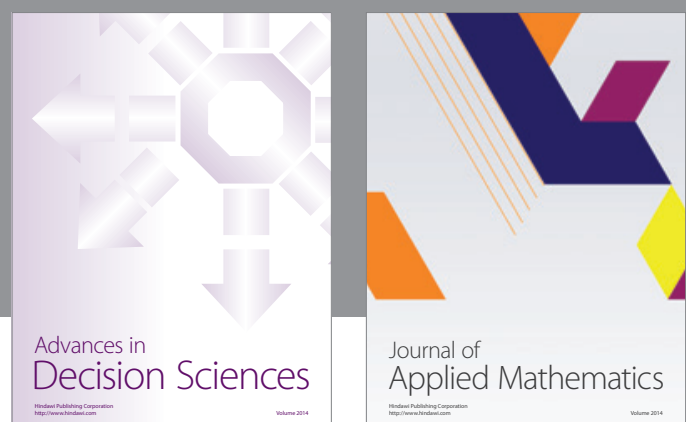

Journal of

Applied Mathematics
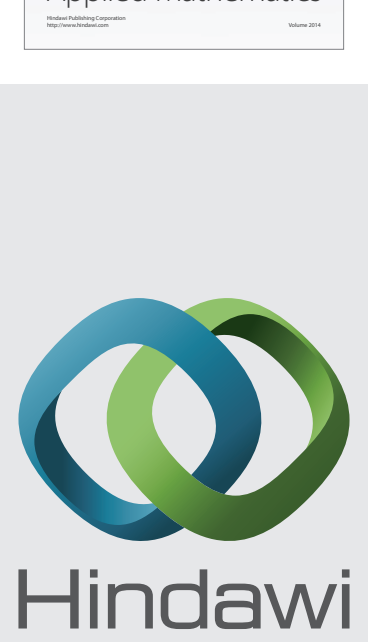

Submit your manuscripts at http://www.hindawi.com
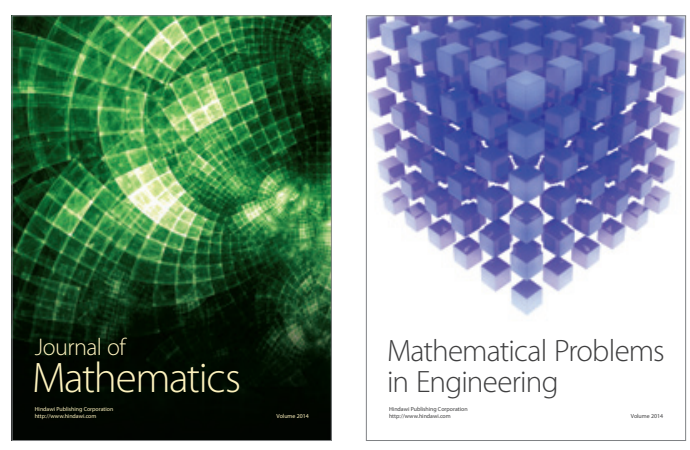

Mathematical Problems in Engineering
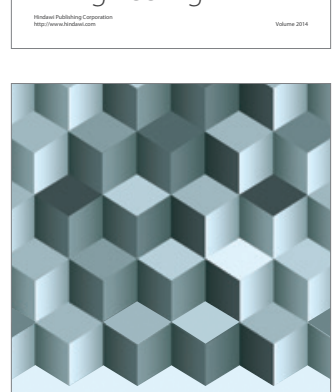

Journal of

Function Spaces
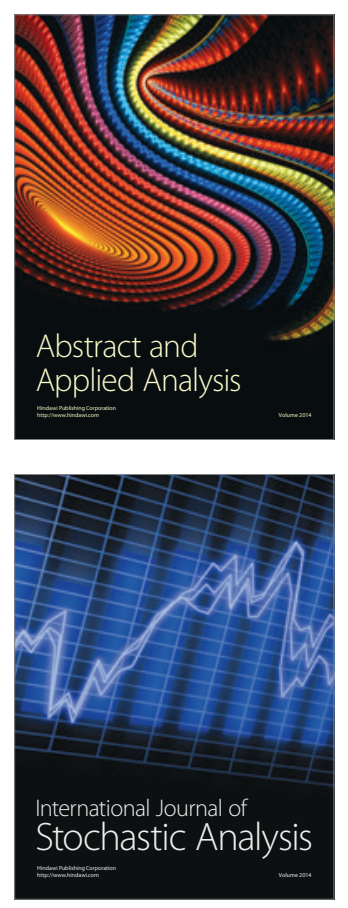

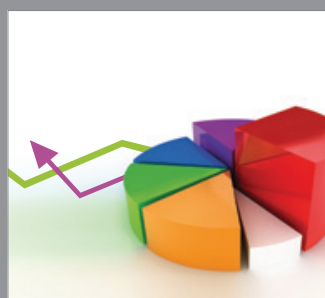

ournal of

Probability and Statistics

Promensencen
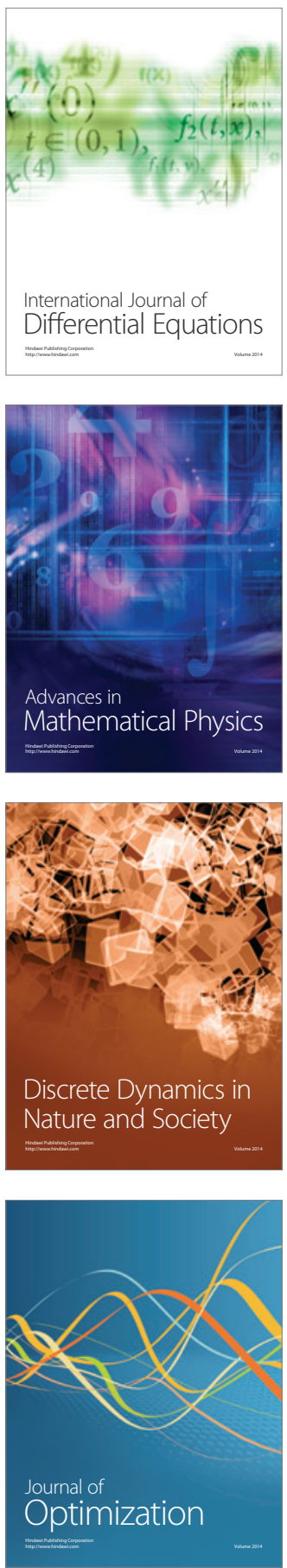